\title{
The mechanical properties of the rubber elastic polymer polydimethylsiloxane for sensor applications
}

\author{
J C Lötters $\dagger$, W Olthuis, P H Veltink and P Bergveld \\ MESA Research Institute, University of Twente, PO Box 217, 7500 AE Enschede, \\ The Netherlands
}

Presented on 21 October 1996, accepted for publication on 9 April 1997

\begin{abstract}
Polydimethylsiloxane (PDMS) is a commercially available physically and chemically stable silicone rubber. It has a unique flexibility with a shear elastic modulus $G \approx 250 \mathrm{kPa}$ due to one of the lowest glass transition temperatures of any polymer $\left(T_{g} \approx-125^{\circ} \mathrm{C}\right)$. Further properties of PDMS are a low change in the shear elastic modulus versus temperature $\left(1.1 \mathrm{kPa}^{\circ} \mathrm{C}^{-1}\right)$, virtually no change in $G$ versus frequency and a high compressibility. Because of its clean room processability, its low curing temperature, its high flexibility, the possibility to change its functional groups and the very low drift of its properties with time and temperature, PDMS is very well suited for micromachined mechanical and chemical sensors, such as accelerometers (as the spring material) and ISFETs (as the ion selective membrane). It can also be used as an adhesive in wafer bonding, as a cover material in tactile sensors and as the mechanical decoupling zone in sensor packagings.
\end{abstract}

\section{Introduction}

Polydimethylsiloxane (PDMS) is a commercially available clean room compatible type of silicone rubber with a wide range of applications. It is currently used, for instance, as the mechanical interconnection layer between two silicon wafers [1], as ion selective membranes on ISFETs [2] and as the spring material in accelerometers [3]. Other possible applications are its use as the top elastomer on a tactile sensor such as that described in [4] without influencing the sensitivity of the device and as flexible encapsulation material in order to mechanically and chemically decouple sensors from their environment [5]. Furthermore, it could be used in sensors with integrated electronics due to its low curing temperature.

Some physical and chemical attributes of PDMS are, compared to other polymers [6], a low glass transition temperature $\left(T_{g} \approx-125^{\circ} \mathrm{C}[6]\right)$, a unique flexibility (the shear modulus $G$ may vary between $100 \mathrm{kPa}$ and 3 MPa [6]), very low loss tangent $(\tan \delta \ll 0.001)$, small temperature variations of the physical constants (except for the thermal expansivity, $\alpha \approx 20 \times 10^{-5} \mathrm{~K}^{-1}$ [7]), high dielectric strength $\left(\sim 14 \mathrm{~V} \mu \mathrm{m}^{-1}\right.$ [7]), high gas permeability, high compressibility, usability over a wide

\footnotetext{
$\dagger$ Tel: +31-53-4892755. Fax: +31-53-4892287. E-mail address: j.c.lotters@el.utwente.nl
}

temperature range (at least from $-100^{\circ} \mathrm{C}$ up to $+100^{\circ} \mathrm{C}$ [8]), low chemical reactivity (except at extremes of $\mathrm{pH}$ ) and an essentially non-toxic nature.

This paper describes, the processing of PDMS, the layer thickness versus spin rate, the variation of its shear modulus $G$ with frequency and temperature and its adhesive strength to polished tungsten (after curing); experimental results are also discussed.

\section{Preparation of the PDMS structures}

The materials used were polydimethylsiloxane PS851 from ABCR [8] ((methacryloxypropyl)methylsiloxane), photoinitiator DMAP (2,2-dimethoxy 2-phenylacetophenone) and TMSM from Aldrich (trimethoxysilylpropylmethacrylate).

One wt\% photo-initiator DMAP (powder) was sprinkled into $1 \mathrm{wt} \%$ xylene and the solution was shaken in a Sarstedt CM-9 machine at $1400 \mathrm{rpm}$ for about $1 \mathrm{~h}$ (the temperature was kept at $60^{\circ} \mathrm{C}$ ). The mixture was not used instantly; it had to be kept overnight. The silicon wafer on which the PDMS was spun was cleaned and wet oxidized. A mixture of $89.5 \%$ toluene, $10 \%$ TMSM and $0.5 \%$ demiwater was heated to $60^{\circ} \mathrm{C}$. The wafers were kept in this mixture for one minute so that the methacryl groups present at the wafer surface became attached to the methacryl 


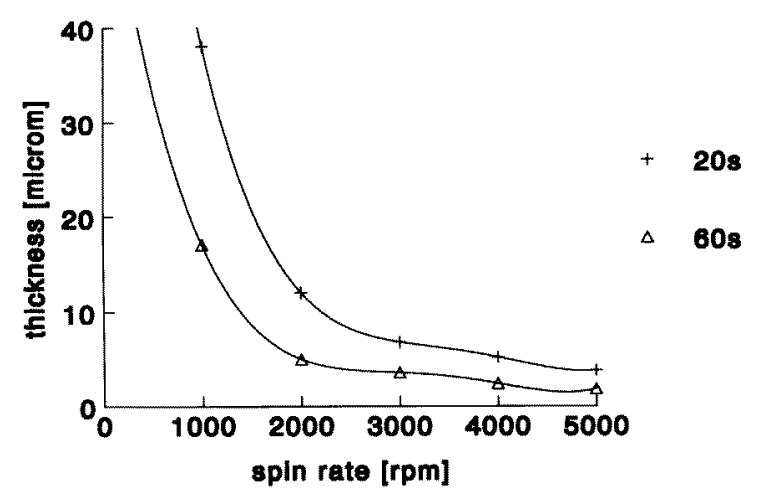

Figure 1. Thickness of polysiloxane PS851 at different spin rates and spin times.

groups of the PDMS. The wafer was rinsed with demiwater to remove surplus TMSM and then was spun dry.

The PDMS was spun upon the wafers with a spin rate varying between 1000 and $5000 \mathrm{rpm}$ and with two spin times, 20 and $60 \mathrm{~s}$. After spinning, the PDMS layer was covered with Mylar foil of $23 \mu \mathrm{m}$ thickness to avoid the presence of oxygen near the PDMS which would disable the cross linking process to occur and to prevent the PDMS sticking to the mask. Thereafter, the PDMS sample was exposed to UV light for $40 \mathrm{~s}$ via a mask and the PDMS which was exposed to the UV light was cross linked. The Mylar foil was then removed and the PDMS was developed in xylene for $30 \mathrm{~s}$, rinsed with isopropanol and spun dry.

\section{Measurement results and discussion}

\subsection{Thickness versus spin rate}

The thickness of the PDMS structures was measured with a DEKTAK II surface profiler with needle radius $250 \mu \mathrm{m}$ and stylus force $0.1 \mathrm{mN}$. The measurement results are shown in figure 1. Thicknesses greater than $40 \mu \mathrm{m}$ can be obtained by applying several layers of PDMS on top of each other.

\subsection{Shear modulus and loss tangent versus frequency and temperature}

A thick cylindrical polysiloxane structure of height $1.2 \mathrm{~mm}$ and radius $8 \mathrm{~mm}$ was fabricated on a silicon substrate to measure the shear modulus $G$. The shear elastic modulus $G$ can be divided into a real part $G^{\prime}$ and an imaginary part $G^{\prime \prime}[8]: G=G^{\prime}+\mathrm{i} G^{\prime \prime}$. The loss tangent $\tan \delta$ is equal to $G^{\prime \prime} / G^{\prime}$.

The variation in $G$ due to changes in frequency and temperature can be measured with the Bohlin rheometer system. The Bohlin can apply frequencies in the range between 0.005 and $30 \mathrm{~Hz}$ and temperatures between $0^{\circ}$ and $70{ }^{\circ} \mathrm{C}$.

The measurement system consists of two parallel circular discs with the polysiloxane structure in between. The lower disc applies a certain torque with a certain frequency to the structure, a torsional force measuring device is connected to the upper disc and this measures the resulting movement of the polysiloxane due to the applied force.
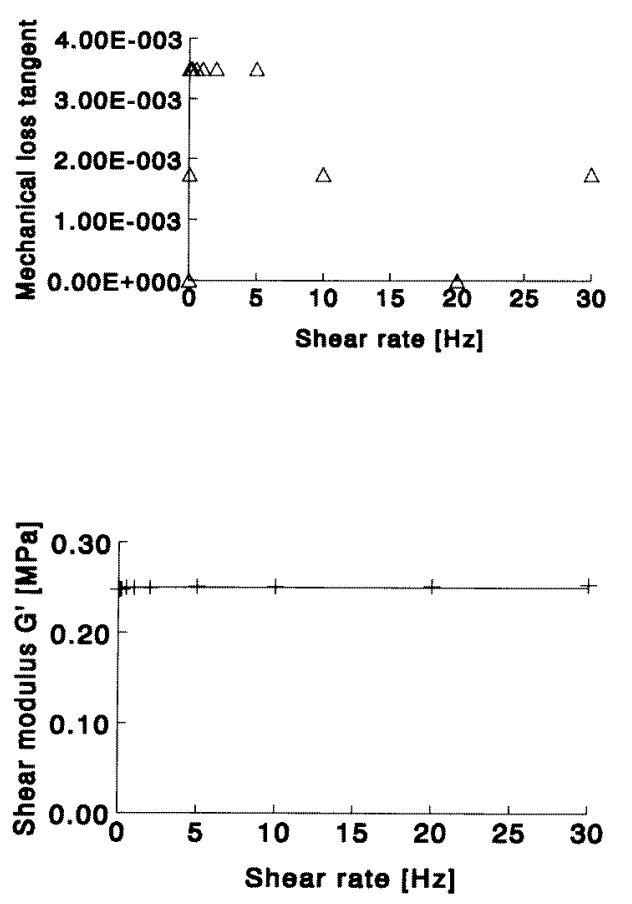

Figure 2. Shear modulus $G^{\prime}$ (bottom) and loss tangent (top) versus shear rate.

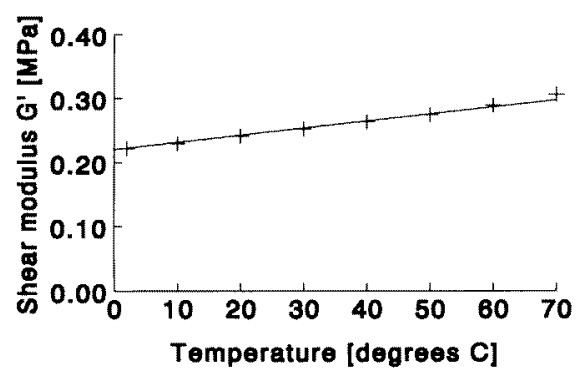

Figure 3. Shear modulus $G^{\prime}$ versus temperature, at frequencies between 0.005 and $30 \mathrm{~Hz}$.

The shear modulus $G^{\prime}$ and the loss tangent $\tan \delta$ versus applied frequency are shown in figure $2 ; G^{\prime}$ versus temperature is shown in figure 3.

Figure 2 shows that the shear modulus is independent of the applied frequency, which is typical for a rubberelastic material. The loss tangent is so small that its value was determined by the accuracy of the equipment rather than by its actual value, so it can be concluded that these are the absolute maximum values. Figure 3 shows that the shear modulus increases with temperature, which is typical for rubberelastic materials at the 'rubbery plateau' [8]. The variation of the loss tangent with temperature was not measured because of the inaccuracy of the equipment used.

\subsection{Adhesion of PDMS to an oxidized silicon wafer}

A primer like TMSM should be used as the coupling agent between an organic polymer like PDMS and a mineral substrate such as oxidized silicon. The primer is able to chemically react with the silicon oxide surface and it contains at least one other functional group that can react with the PDMS during curing (the methacrylate group in 
the case of TMSM). In this way the primer acts as a linker to bind the silicon oxide surface to the PDMS covalently [5]. The adhesion between the silicon oxide and the PDMS is very strong-it was not possible to separate the PDMS from the wafer by manual peel tests.

\subsection{Adhesion of cured PDMS to polished tungsten}

The surface forces for elastomers which are responsible for adhesion between a cured elastomer and a rigid smooth surface can arise from (1) van der Waals forces, (2) electrostatic forces and (3) hydrogen bonds. It is not clear which forces provide the major source of bonding, but most observations are in favour of the van der Waals forces. The more flexible the polymer and the less rough the rigid solid surface on which the cured polymer is put, the better the adhesion: when the average surface roughness is less than $0.33 \mu \mathrm{m}$ and $G \leq 250 \mathrm{kPa}$ the relative adhesion is higher than $50 \%$ [9]. To test the adhesive strength, polished tungsten cubes of $3 \times 3 \times 3 \mathrm{~mm}^{3}$ with a mass of $520 \mathrm{mg}$ and an average surface roughness of $0.3 \mu \mathrm{m}$ were placed upon several PDMS structures with areas varying from $4.7 \times 10^{-8} \mathrm{~m}^{2}$ up to $4.7 \times 10^{-7} \mathrm{~m}^{2}$ and accelerations up to $160 \mathrm{~m} \mathrm{~s}^{-2}$ were applied to the constructions with a Gearing and Watson GWV20 shaker unit. Thus, adhesive strengths up to $180 \mathrm{kPa}$ were observed.

\section{Conclusions}

Polydimethylsiloxane is the most flexible polymer with a shear modulus $G \approx 250 \mathrm{kPa}$ at room temperature. The shear modulus is independent of the applied frequency but linearly dependent on the temperature with a slope of $1.1 \mathrm{kPa}{ }^{\circ} \mathrm{C}^{-1}$. The loss tangent $\tan \delta$, which has a value $\tan \delta \ll 0.001$ according to the literature, could not be determined due to the inaccuracy of the equipment used. The results show that PDMS is a rubberelastic material. For rubberelastic materials, Young's modulus $E \approx 3 G$, so here $E \approx 750 \mathrm{kPa}$.

When a primer is used, a very good adhesion is obtained between oxidized silicon and PDMS. Furthermore, due to the low surface energy and high flexibility of PDMS a good adhesion is obtained between cured PDMS and polished surfaces with an average roughness of less than $0.33 \mu \mathrm{m}$. Adhesive strengths up to $180 \mathrm{kPa}$ were observed in this case.
PDMS is commercially available with a selection of functional groups which allows various curing schemes to enable patterning, bonding and chemical selectivity. Apart from known applications as mechanical interconnection layers between two silicon wafers, ion selective membranes on ISFETs and spring material in accelerometers, new applications for PDMS could be a flexible top elastomer in tactile sensors (without influencing the sensitivity of the device) and a flexible encapsulation material in order to mechanically and chemically decouple sensors from their environment.

\section{Acknowledgment}

The authors would like to thank $\mathrm{Mr} \mathrm{J}$ G Bomer and $\mathrm{Mr}$ A $\mathbf{J}$ Verloop for their assistance in device preparation and the Dutch Technology Foundation (STW) for its financial support.

\section{References}

[1] Arquint P, van der Wal P D, van der Schoot B H and de Rooij N F 1995 Flexible polysiloxane interconnection between two substrates for microsystem assembly Proc. Transducers '95 (Stockholm) 1995 (Stockholm: Foundation for Sensor and Actuator Technology) pp 263-6

[2] Antonisse M M G, Engbersen J F J and Reinhoudt D N 1995 Nitrate and bicarbonate selective CHEMFETs Proc. Transducers '95 (Stockholm) 1995 (Stockholm: Foundation for Sensor and Actuator Technology) pp 867-9

[3] Lötters J C, Olthuis W, Veltink P H and Bergveld P 1996 Polydimethylsiloxane as an elastic material applied in a capacitive accelerometer J. Micromech. Microeng. 6 $52-4$

[4] Chu Z 1996 Flexible package for a tactile sensor array Proc. 1996 National Sensor Conf. (Delft, The Netherlands) March 20-21 1996 (Delft: Delft University Press) pp 121-4

[5] van Hal R E G 1994 Advanced packaging of ISFETs, design, encapsulation and bonding PhD Thesis University of Twente

[6] Clarson S J and Semlyen J A 1993 Siloxane Polymers (Englewood Cliffs, NJ: Prentice-Hall)

[7] ABCR 1994 Research Chemicals and Metals Catalogue Karlsruhe, Germany

[8] van Krevelen D W and Hoftyzer P J 1976 Properties of Polymers (Amsterdam: Elsevier)

[9] Clark D T and Feast W J 1978 Polymer Surfaces (New York: Wiley) 